\title{
Effects of Integrative Chinese and Western Medicine on Arterial Oxygen Saturation in Patients with Severe Acute Respiratory Syndrome*
}

\author{
LIU Bao-yan(刘保延 $)^{1}$, HU Jing-qing(胡镜清 $)^{1}$, XIE Yan-ming(谢雁鸣 $)^{1}$, WENG Wei-liang(翁维良 ${ }^{2}$, \\ WANG Rong-bing (王融冰) ${ }^{3}$, ZHANG Yan-ping(张燕萍) ${ }^{2}$, LI Xiu-hui(李秀惠 $)^{4}$, ZHANG Ke(张 可) ${ }^{4}$, \\ REN Ai-ming (任爱民) $)^{5}$, LI Jun(李 筑) ${ }^{6}$, WANG Bao-guo(王保国) ${ }^{7}$, TANG Xu-dong(唐旭东) ${ }^{8}$, \\ WANG Wei-dong (汪卫东) $)^{8}$, NI Qing(倪 青) ${ }^{8}$, ZHANG Jin-ping(张进萍) ${ }^{9}$, WU Hong-jin(吴红金) $)^{10}$, \\ ZHOU Wei(周 卫 $)^{11}$, GENG Zhi(耿 直) ${ }^{12}$, HE Yang-bo(何洋波 $)^{12}$, LIANG Zhi-wei(梁志伟) ${ }^{13}$,

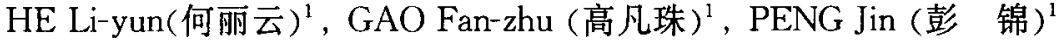

\begin{abstract}
Objective: To evaluate the effects of treatment of integrative Chinese and Western medicine (ICWM) on arterial oxygen saturation $\left(\mathrm{SaO}_{2}\right)$ in patients with severe acute respiratory syndrome (SARS). Methods: The non-randomized, controlled trial was conducted on 447 SARS patients treated synchronously with western conventional treatment (WM group, $n=171$ ) alone and ICWM (ICWM group, $n=276$ ). The changes of the cases with normal level $(\geqslant 95 \%)$ or abnormal level $(<95 \%) \mathrm{SaO}_{2}$ were observed dynamically. Results: In the 3rd-14th day of the therapeutic course, the percentage of patients with normal $\mathrm{SaO}_{2}$ in the ICWM group was higher than that in the WM group (OR $=0.5178, P=0.0038)$, and this tendency was more evident in patients of the severe type $(O R=0.18, P=0.0001)$. However, the statistical significance of difference was only shown in patients for whom the ICWM treatment started in the early period after the onset $(\leqslant 7$ days after it, $O R=0.3803, P=0.006)$, but not shown in those who received ICWM treatment later in the mid-late period of SARS $(P>0.05)$. Conclusion: ICWM treatment, particularly when it is used for intervention in the early stage, is beneficial for maintaining normal $\mathrm{SaO}_{2}$ in SARS patients.

KEY WORDS severe acute respiratory syndrome, arterial oxygen saturation, integrative Chinese and Western medicine
\end{abstract}

Apparent lowering of blood oxygen saturation $\left(\mathrm{SaO}_{2}\right)$ induced by acute pulmonary injury is an important clinical manifestation of acute severe respiratory syndrome (SARS). In order to verify the efficacy of integrative Chinese and Western medicine (ICWM) in treating SARS, the dynamic changes in arterial $\mathrm{SaO}_{2}$ of the ICWM treated group and simple Western medicine (WM) treated group were analyzed to understand the possibly existing difference.

\section{METHODS}

\section{Clinical Materials}

The subjects enrolled in this study were SARS patients, whose diagnosis fit to the clinical diagnostic criteria of severe acute respiratory syndrome (SARS) issued by the Ministry of Health ${ }^{(1)}$ and agreed with the trial. Those with unconfirmed diagnosis, or pregnancy or lactation, or who refused to participate in the observation were excluded.
Eight hospitals designated for SARS treantment in various districts of Beijing were chosen as the clinical observation units. Based on their own condition, each unit selected for observation patients who were hospitalized from May, 2003 to June, 2003 in wards with similar condition and met the above-mentioned criteria for inclusion. They were assigned into the $\mathrm{WM}$ group, receiving

\footnotetext{
* This item is one of the items of State 863 Plan (No. 2003AA208101); a special subject of clinical study of State administration of TCM; and an important item of Beijing Scientific-Technology Plan(No. H020920010031)

1. Clinical Evaluation Center, China Academy of TCM; 2. Xiyuan Hospital, China Academy of TCM; 3. Beijing Municipal Ditan Hospital; 4. Beijing Municipal You'an Hospital; 5. Beijing Friendship Hospital; 6. 302 Hospital, PLA; 7. Beijing Tiantan Hospital; 8. Guang' anmen Hospital, China Academy of TCM; 9. Beijing Municipal Chest Hospital. 10. Beijing Municipal Hospital of Integrative Traditional Chinese and Western Medicine; 11. Wangjing Hospital, China Academy of TCM; 12. Peking University; 13. Guangdong Provincial Hospital of TCM

Correspondence to:Dr. LIU Bao-yan

Tel:010-6401441 Ext. 2213

E-mail: liuby@mail.cintcm.ac.cn
} 
conventional treatment of WM alone, and the ICWM group, treated with conventional WM treatment supplemented with Chinese medicine.

In the 549 patients collected, of the 102 patients who were excluded, 14 were due to the $\mathrm{di}^{-}$ agnosis was inconsistent to SARS diagnostic criteria, 11 due their incomplete material of accumulated observation time $<10$ days because of missing in follow-up or transffering to other hospital, and 77 due to the lack of any record on $\mathrm{SaO}_{2}$ in the foremost 26 days after hospitalization. Therefore 447 cases remained were included and analyzed.

The age, sex and illness type, and stage of disease were listed in detail in Table 1. It was shown that at the time of enrolment, the difference between the two groups was significant $(P<$ $0.05)$ in the aspects of age and stage of disease, but insignificant in the aspects of sex and illness type $(P>0.05)$.

Table 1. Comparison of Basic Data between Groups

\begin{tabular}{lcc}
\hline & $\begin{array}{c}\text { ICWM group } \\
(n=276)\end{array}$ & $\begin{array}{c}\text { WM group } \\
(n=171)\end{array}$ \\
\hline $\begin{array}{l}\text { Age } \\
\text { (year, median value) }\end{array}$ & 35.6 & 37 \\
$\begin{array}{l}\text { Sex(F/M) } \\
\text { Type of Illness } \\
\text { (Cases (\%) }\end{array}$ & $152 / 124$ & $92 / 79$ \\
$\quad$ Common type & $169(61.2)$ & $119(69.6)$ \\
$\quad$ Severe Type & $107(38.8)$ & $52(30.4)$ \\
Disease Course (day) & & \\
$\quad \leqslant 7$ & $65(23.6)$ & $57(33.3)$ \\
$\quad 8-14$ & $98(35.5)$ & $62(36.3)$ \\
$>14$ & $113(40.9)$ & $52(30.4)$ \\
\hline
\end{tabular}

Note: critical severe type included in the severe type

\section{Treatment}

WM conventional treatment, referring to the SARS treatment regimen ${ }^{(2)}$ recommended by the Ministry of Health, was given to all patients in both groups, with also supporting therapy given, including respiratory supporting and corticosteroid (CS) treatment.

The mode and parameters of respiratory supporting used were: For patients of the common type, oxygen inhalation was given by routine nasal tube or mask, with a minimum flow of $3-5 \mathrm{~L} / \mathrm{min}$, concentration of the oxygen around $33 \%-100 \%$; for patients of the severe type, non-invasive mechanical ventilation was used, with respirator facial (nasal) bi-level positive airway pressure (BiPAP), an oxygen minimum flow of $5 \mathrm{~L} / \mathrm{min}$, inspiratory positive airway pressure (IPAP) beginning from $8 \mathrm{~cm} \mathrm{H}_{2} \mathrm{O}$, the scale in $10-20 \mathrm{~cm} \mathrm{H}_{2} \mathrm{O}$, and expiratory positive airway pressure (EPAP) $4-10 \mathrm{cmH}_{2} \mathrm{O}$.

CS treatment was given according to the condition of pulmonary inflammation, using mainly the methylprednisolone, with a daily dosage of $80-160 \mathrm{mg}$, in severe cases, it might went up to $320-400 \mathrm{mg}$, or even the maximum of $800-$ $1000 \mathrm{mg}$.

TCM treatment regimen was used on the basis of Syndrome Differentiation, which refers to the "Technical Regimen of Chinese Medicine in Treating and Preventing SARS" (draft) issued by State Administration of Traditional Chinese Medicine.

For patients in the early stage $(\leqslant 7$ days after onset), when the Syndrome type was of HeatToxin evil invading Fei (肺), they should be treated with the principle of clearing Heat and ventilating Fei, dispersing the exterior and emancipating the Collateral, using modified Yinqiao powder (银 趐散) combined with Maxing Shigan decoction (麻 杏石甘汤); when the Syndrome type was of HeatDamp obstruction, the principle of ventilating Damp-Heat, and driving evil pathogen outward was used with modified Sanren decoction (三仁汤) combined with Shengjiang powder (升降散); when Damp is severer than Heat, Huopu Xialing decoction (嚄朴夏苓汤) could be chosen for use; when the Syndrome type was of Cold exterior-heat interior complicated with Damp, the principle of resolving Exterior and clearing internal Heat and dispersing Fei to remove Damp was used with modified Maxing Shigan decoction (麻杏石甘汤) combined with Shengjiang powder (升降散).

For patients in the middle stage ( $8-14$ days after onset), when the Syndrome type was of epidemic Toxin invading Fei and Heat exuberance in both exterior and interior, the principle of clearing Heat and relieving toxicity and purging Fei to lower reversed flow of Qi was used with Qingfei Jiedu decoction (清肺解毒汤); when Syndrome type was of Damp-Heat bound by Toxin, the principle of removing Damp, depriving foul, clearing Heat and relieving toxicity was used with modified Ganlu Xiaodu pill (甘露消毒丹); when Syndrome type was of accumulation of Damp-Heat obstructing Shaoyang meridian, the principle of clearing and purging Shaoyang meridian and eliminating Damp and Heat was chosen with modified Haoqin Qing- 
dan decoction (蒿芩清胆汤); when the Syndrome type was of exuberant Heat-toxin, the principle of clearing Heat, cooling blood, purging Fire and relieving toxicity was chosen with modified Qingwen Baidu decoction (清瘟败毒汤).

For patients in the late stage (usually around 14 days after onset), when the Syndrome type was of Phlegm-Damp with Toxin stagnancy obstructing Fei Collateral, the principle of replenishing Qi, relieving toxicity, removing Phlegm and Damp, cooling blood and dredging Collateral was chosen with Huoxue Xiefei decoction (活血泻肺 汤); when Syndrome type was of Damp-Heat obstructing Fei with both Qi-Yin damage, the principle of clearing Heat, removing Damp, tonifying Qi and nourishing Yin was chosen with Yifei Huazhuo decoction (益肺化浊汤); when Syndrome type was of evil prominence with vital Qi asthenia, and interior blockage with asthma collapse, the principle of replenishing $\mathrm{Qi}$ to consolidate collapse, and dissolving blockage to open up the orifices was chosen with modified Shenfu decoction (参附汤).

For patients in the rehabilitation stage $(\geqslant 15$ days after onset), when Syndrome type was of QiYin damage with residual evil, the principle of replenishing Qi to nourish Yin, and removing Damp to free Collateral was chosen with modified Li's Qingshu Yiqi decoction (李氏清暑益气汤); when Syndrome type was of Fei-Pi deficiency, the principle of replenishing $\mathrm{Qi}$ and activating $\mathrm{Pi}$, was applied with modified Shenling Baishu powder (参苓 白术散) combined with Gegen Qinlian decoction (葛根芩连汤).

The dosage form of Chinese herbal medicine used was mainly decoction, though sometimes injections and Chinese patent drugs could be selected.

\section{Items and Methods of Observation and Analy- sis}

1. Analysis of dynamic changes of $\mathrm{SaO}_{2}$ after treatment in SARS patients

$\mathrm{SaO}_{2}$ was detected by taking $1 \mathrm{ml}$ of arterial blood, which was anticoagulated with small amount of heparin, and isolated from air, and immediately sent into blood-gas analyzer for examination. $\mathrm{SaO}_{2} \geqslant 95 \%$ was regarded as normal, $<$ $95 \%$ as abnormal.

After the data were adjusted regarding such factors as age, type and stage of disease, treat- ment course, the difference of $\mathrm{SaO}_{2}$ in different stages (early, middle and late) was compared, and that in different treatment courses and disease types was analyzed level by level through model setting. Observation was performed in the period from the hospitalization of patients till their clinical improvement or dischargs, death or dropping out from the follow-up due to transferring to other hospital.

2. Condition of respiratory support and CS administration

\section{Data Management and Statistical Analysis}

Case report forms about changes in conditions of disease and treatment measures were filled in time by the doctor in charge in various hospitals (observation units) which was faxed to the data management center every day, where the completeness and correctness of data was confirmed through form checking, error adjusting, source data verification and correction. Then entry of the data were made by two persons separately into the database created by Microsoft Access 2000 for statistical analysis by software SPSS 12.0 through data check (logic and scope) and correction.

The sorted-out data frequency was analyzed with $\chi^{2}$ test or rank sum test, aSbnormal distribution measurement data analyzed with rank sum test, and the difference of $\mathrm{SaO}_{2}$ between groups compared with generalized linear mixed model es tablished by SAS 6.12 software package.

\section{Ethic Question and Consent}

The research protocol was approved by the ethic committee of Guang'anmen Hospital, China Academy of TCM, and all the enrolled patients had been informed about the test and consented to it orally, accepting respectively the ICWM or simple WM treatment regimen.

\section{RESULTS}

\section{Evaluation Criteria}

The "Discharging criteria of SARS" (3) issued by the Ministry of Health was taken for evaluating clinical improvement and used as the basis for the discharge.

\section{Comparison of Respiratory Support Treatment and CS Usage}

See Table 2. The difference between the two groups in number of patients who 
Table 2. Comparison in Respiratory Support Used and CS Administration between the Two Groups

\begin{tabular}{|c|c|c|c|c|c|c|}
\hline \multirow{2}{*}{ Group } & \multirow{2}{*}{$n$} & \multicolumn{2}{|c|}{ Oxygen Inhalation } & \multicolumn{2}{|c|}{ Machine Application } & \multirow{2}{*}{$\begin{array}{l}\text { Dosage of CS used } \\
\text { (median value, } \mathrm{mg} \text { ) }\end{array}$} \\
\hline & & Time (day, $\bar{x} \pm s)$ & Cases & Time (day, $\bar{x} \pm s$ ) & Cases & \\
\hline ICWM & 276 & $10.0 \pm 5.4$ & 213 & $7.9 \pm 4.6$ & 57 & 1045 \\
\hline WM & 171 & $10.0 \pm 4.8$ & 136 & $8.7 \pm 5.3$ & 47 & 1200 \\
\hline$P$ value & & 0.997 & 0.5581 & 0.46 & 0.0722 & 0.428 \\
\hline
\end{tabular}

used nasal tube or mask oxygen inhalation and in total amount of CS administered was insignificant $(P>0.05)$. The percentage of patients using breathing machine in the ICWM group was $20.7 \%(57 / 276)$, while that in the WM group was $27.5 \%(47 /$ 171 ), showing an increasing tendency in the WM group as compared with that in the ICWM group ( $P=0.072)$, but no statistical significance was shown. The dosage of CS used between the two groups showed no significant difference.

\section{Dynamic Change of $\mathrm{SaO}_{2}$ after Treatment}

As shown in Fig. 1. There was insignificant difference in $\mathrm{SaO}_{2}$ level (expressed by the percentage of patients with abnormal $\mathrm{SaO}_{2}$ level, abbreviated as PPS) between the two groups during the whole treatment course $(P=0.088)$. Further comparison through model creating and stratification indicated that at the baseline stage of $0-2$ days, there was insignificant difference in comparison of PPS between the two groups $(P=0.4464)$, but after being treated for $3-14$ days, the PPS in the ICWM group was lower than that in the WM group ( $\mathrm{OR}=0.5178, P=0.0038$ ), and this tendency was more evident in patients of the se-

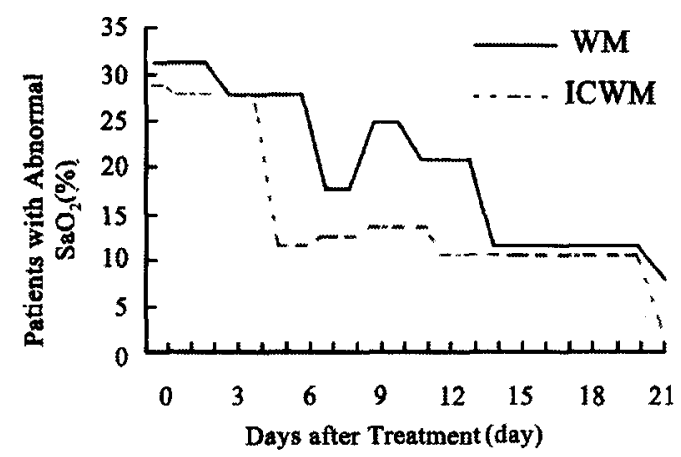

Fig. 1 Dynamic Changes of $\mathrm{SaO}_{2}$ of Two Groups vere type $(\mathrm{OR}=0.18, P=0.0001)$. Moreover, comparison of PPS between the two groups after 15 or more days of treatment (late stage) also showed significant difference $(\mathrm{OR}=0.2426, P=0.0007)$.

There was insignificant difference in terms of lowering the risk of abnormal $\mathrm{SaO}_{2}$ level in the common type of patients between the two groups ( $\mathrm{OR}=1.22, P=$ 0.4745 ), but as far as patients of the severe type were concerned, comparison between the two groups did show a better therapeutic effect in the ICWM group than that in the $\mathrm{WM}$ group $(\mathrm{OR}=0.18, P=0.0001)$.

\section{Comparison of PPS between the Two Groups in Patients Starting the Treatment at Various Stages}

The PPS in the patients of the two groups on whom the treatment started at early stage ( $\leqslant 7$ days after onset) was shown in Fig 2, which indicated that the condition was obviously better in the ICWM group than that in the WM group within the 3rd14 th day of the therapeutic courset, showing significant difference $(\mathrm{OR}=0.3803, P=$ $0.006)$.

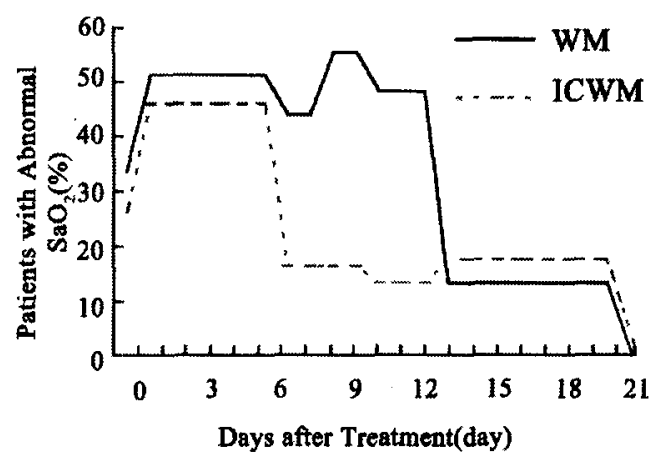

Fig. 2 Comparison of Dynamic Changes of PPS in the Early Intervention of the Two Groups 
However, the difference was insignificant in the course before the $3 \mathrm{rd}$ day or after the 15 th $\operatorname{day}(\mathrm{OR}=0.1356, P=0.1282)$.

As for patients, on whom the treatment started at the middle ( $8-14$ days after onset) or late ( $\geqslant 15$ days after onset) stage, insignificant difference was shown in comparisons of PPS between the two groups, either in total course or in different partial courses of treatment. See Fig 3 and 4 .

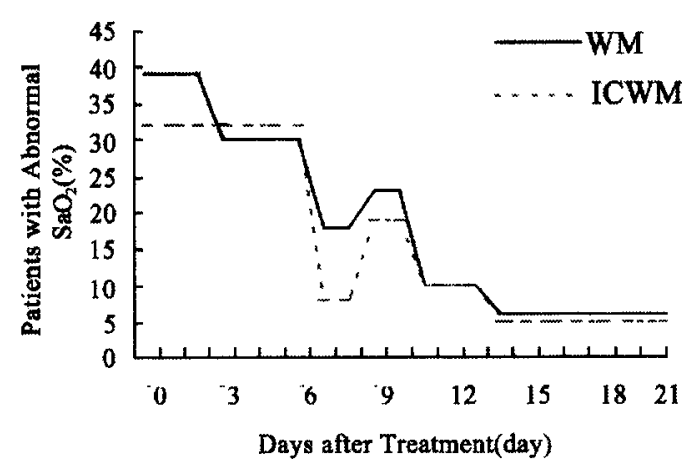

Fig. 3 Comparison between Dynamic Changes of PPS in the Middle Intervention of the Two Groups

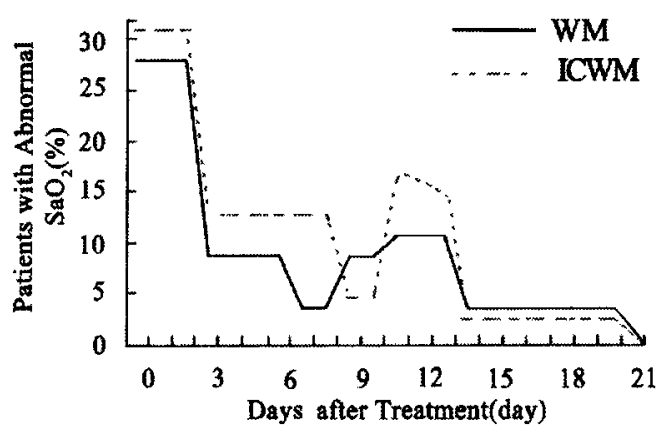

Fig. 4 Comparison between Dynamic Changes of PPS in the Late Intervention of the Two Groups

\section{DISCUSSION}

The present study has proved that the manifestations of SARS are acute diffused pulmonary damage and adult respiratory distress syndrome (ARDS), and the pathological mechanism is mainly the reproduction of virus-caused respiratory mucous membrane epithelial and alveolar cell necrosis with immune injury simultaneously. The secondary immune injury is considered to be a possible key link of pulmonary damage of $\mathrm{SARS}^{(4)}$. The products produced after mucous membrane epithelial cell and alveolar cell necrosis would further stimulate tissues to produce inflammatory medium, which induces increase of alveolar septum capillary permeability, so as to cause inflammatory exudation, and then result in widening of alveolar septa, enlarging air-blood barrier interstitial space, and finally leading to ventilation disturbance.

When the disease progresses further, the concentrated increased exudation forms a homogenous transparent membrane, and stick to the alveolar wall, thus to aggravate air exchange disturbance. At the same time, large amount of exudative fluid can fill the bronchial lumen, cause obstruction to aggravate ventilation disturbance, and induce serious hypoxia of organism.

At present, the standard therapeutic agents for curing SARS include antiviral agent, glucocorticoid, antibiotics, immunomodulators, etc. Although the majority of specialists in clinical medicine, basing themselves on their therapeutic experience in treating similar diseases, held that these agents are beneficial in treating SARS, there still lacks convincing evidence ${ }^{(5-9)}$.

Studies indicated that ICWM treatment with Chinese herbal medicine added is helpful in improving patients' symptoms, recovering their immune functions, etc. ${ }^{(10-12)}$. But, no report on the effect about $\mathrm{SaO}_{2}$ has been seen. In this study, there were some problems regarding the obtained $\mathrm{SaO}_{2}$ data owing to the limitations in conditions of study, such as the incongruent time point of $\mathrm{SaO}_{2}$ measuring, and the unbalancing factor brought about by non-randomization. And at the same time, with the same patient checked at different time points, there must exist the problem of imbalance and repeated data with different time span. The generalized linear mixed model in SAS software package was used in the present study to es- 
tablish longitudinal data logistic model, and the influencing factors were regulated, so as to well resolve these problems.

It was found in the study that when the treatment was combined with the intervention therapy with Chinese herbal medicines, i. e. when ICWM was used for 3 days, there were more patients who recovered the ability of maintaining $\mathrm{SaO}_{2}$ level than those treated with WM alone, which was more obvious in patients of the severe type. Further analysis indicated that in patients treated with early intervention ( $0-7$ days) of Chinese medicine, the difference in efficacy between the ICWM and WM groups was significant, while if the intervention treatment was applied in the middle or late stage ( 8 days after onset), the difference was insignificant. The above-mentioned results indicated that ICWM treatment could evidently improve the $\mathrm{SaO}_{2}$ in patients, and the efficacy of early intervention could yield better results.

Some deficiencies existed in the present study. For example, the study was originally designed as a randomized controlled trial, but owing to the limitations of conditions, not all the observed cases underwent a fully randomized distribution. At the same time, no record was made about part of the cases, which caused incomplete data and made the study liable to potentially deviated selection, and therefore the conclusion needs to be further studied and confirmed.

\section{REFERENCES}

1. Ministry of Health, People's Republic of China. The clinical diagnostic criteria of SARS 2003; May, 3.

2. Ministry of Health, Peoplés Republic of China. The recommended treatment regimen of SARS. 2003; May, 3.

3. Ministry of Health, People's Republic of China. The dis ${ }^{-}$ charging referential criteria of SARS patients 2003; May, 3.

4. Nicholls JM, Poon LM, Lee KC, et al. Lung pathology of fatal severe acute respiratory syndrome. Lancet 2003; $361: 773-1778$.

5. Oba Y. The use of corticosteroids in SARS. N Engl J Med 2003; 348 : 2034-2035.

6. Liu ZY, Li TS, Wang Z, et al. Clinical characteristics and treatment summarization of 106 SARS patients. Chin J Int Med 2003; 42(6) : 373-377.

7. Peiris JS, Chu CM, Cheng VC, et al. Clinical progression and viral load in a community outbreak of coronavirus associated SARS pneumonia: a prospective study. Lancet 2003; $361: 1767-1772$.

8. Bermejo Martin JF, Jimenez JL, Munoz Fernandez A. Pentoxifylline and SARS: a drug to be considered. Med Sci Monit 2003; $9:$ SR29-34.

9. So LK, Lau AC, Yam LY, et al. Development of a standard treatment protocol for SARS. Lancet 2003; $361: 1615-1617$.

10. Lin L, Han Y, Yang ZM, et al. Clinical observation on 103 SARS patients with integrative Chinese and western medicine. Chin J Integr Tradit West Med 2003; 23 (6) : 409-413.

11. Li XH, Zhang $\mathrm{K}, \mathrm{Hu} \mathrm{JH}$, et al. Clinical observation of efficacy of integrative Chinese and western medicinein treating SARS. Chin J Integr Tradit West Med 2003; 23 (7) : 489-491.

12. Wang RB, Liu AM, Jiang YB, et al. Preliminary analysis on efficacy of integrative Chinese and western medicine in treating SARS. Chin J Integr Tradit Wes Med $2003 ; 23(7): 492-493$

(Received March 1, 2004)

\section{Clinical Observation on Effect of Xuesaitong Injection (血塞通注射液) as Auxiliary Treatment of Severe Craniocerebral Injury}

AI Wen-bing(艾文兵), CHEN Yu-hong(陈玉宏), and YANG Qi-jian(杨启建)

Department of Neurosurgery, Affiliated Renhe Hospital of Three Gorges University, Hubei (443001)

Objective To evaluate the effect of Xuesaitong injection (XSTI, 血塞通注射液, a preparation of Panax Notoginseng) as auxilliary treatment of severe craniocerebral injury. Methods Eightyseven patients with severe craniocerebral injury were selected and randomly divided into the treated group $(n=44)$ and the control group $(n=43)$, they were treated with conventional treatment, and XSTI was given additionally to the treated group. Intracranial pressure (ICP) and Glasgow coma score (GCS) of all patients were measured after 1 or 2-week treatment, and Glasgow outcome scale (GOS) of them was determined 3 months later. Then the therapeutic effect in the two groups were compared. Results After treatment, the ICP was lower, GCS higher and GOS better in the treated group than those in the control group significantly, all showing statistical significance $(P<0.05)$. Conclusion XSTI has marked clinical therapeutic effect in treating patients with severe craniocerebral injury.

Original article on CJITWM (Chin) $2004 ; 23(3): 213$ 